\title{
Les causes d'échec du management africain
}

\author{
Chirushage Chirishungu $^{1}$ \\ Université du Q uébec à Chicoutimi
}

Pourquoi avons-nous des problèmes de gestion en Afrique? Les raisons avancées pour expliquer les déficiences de la gestion africaine sont liées soit à l'histoire, soit à la conjoncture, soit à l'instabilité politique, soit à des pratiques de gestion inadéquates. Deux des principales causes sont d'ordres culturel et environnemental. La première rejoint le fait que les valeurs africaines sont peu ouvertes à l'adoption de nouvelles pratiques de gestion. Ces valeurs se reflètent tant dans la structure et dans la forme des organisations que dans les aspirations et comportements des individus qui y travaillent. Ceci rend la cohabitation du monde moderne et du monde traditionnel difficile, car chacun possède son propre mode de fonctionnement. La deuxième cause est liée à l'incertitude et aux changements constants qui caractérisent l'environnement des entreprises, qui font que les gestionnaires africains renoncent à planifier alors que l'application de certains processus managerials flexibles pourrait amoindrir cette incertitude. L'apport des pratiques de gestion en matière d'efficacité organisationnelle a été prouvé. Il n'y a aucun doute que les entreprises africaines ont besoin de recourir à certaines techniques manageriales pour améliorer leur efficacité. Le défi posé consiste donc à trouver le moyen de les adapter au contexte africain, c'est-à-dire de les rendre compatibles aux valeurs africaines. L'urgence s'impose quant à la résolution de ce problème, et le plus tôt serait le mieux car en ce moment même, tandis que certains auteurs estiment que le management d'hier ne fonctionne plus et que les entreprises occidentales sont à la recherche de l'organisation de demain, les entreprises africaines tentent de s'ajuster au management d'hier.
Les études réalisées au Niger, au Burundi et au Rwanda sur les facteurs susceptibles d'influencer les pratiques de gestion dans les entreprises, à savoir les caractéristiques du propriétaire-dirigeant, de l'entreprise et l'environnement organisationnel, ont permis d'obtenir les résultats suivants : dans ces pays, la planification se fait de façon sporadique, informelle et dans la plupart des cas, intuitive. Au Niger, l'organigramme est considéré comme indispensable dans les relations internes de l'entreprise. Par contre, au Rwanda et au Burundi, il n'est pas perçu comme un outil de gestion en soi. Il est le plus souvent établi au moment des formalités administratives à l'étape de la création de l'entreprise ou lors d'une demande de crédits et est ensuite oublié. Les entreprises semblent faire un usage étendu de la plupart des techniques de contrôle au Burundi et au Rwanda, mais au Niger, le système de contrôle en est au stade embryonnaire. Les résultats d'ensemble obtenus révèlent que dans ces pays, plus de $50 \%$ des entreprises n'ont pas recours aux pratiques de gestion. L'analyse des facteurs environnementaux indique que l'environnement organisationnel est peu favorable au développement du secteur privé. Les problèmes le plus souvent rencontrés sont le manque de disponibilité des ressources matérielles, des ressources financières, des ressources humaines qualifiées ainsi que les formalités administratives avant et après la création de l'entreprise.

Une étude similaire a été réalisée en République démocratique du Congo auprès de 34 entreprises de la ville de Kinshasa. Les résultats ont révélé que les caractéristiques des propriétaires-dirigeants n'influencent pas l'utilisation des outils de gestion, bien que sur les $58 \%$ ayant fait des études universitaires, $35 \%$ ont 
suivi une formation en gestion. L'analyse sur l'environnement indique que $85 \%$ des propriétairesdirigeants considèrent que les institutions financières en place ne répondent pas au besoin en capital des agents économiques. Du total des propriétairesdirigeants, $24 \%$ seulement ont bénéficié d'un crédit bancaire lors de la création de l'entreprise - ce qui reflète fort bien l'état actuel du réseau bancaire congolais - et $26 \%$ ont bénéficié d'un programme étatique ou privé de soutien aux entreprises. Quant à la performance, elle a été déterminée selon le critère de profitabilité. Les résultats obtenus ont montré que de l'ensemble des entreprises retenues, $24 \%$ sont performantes, $41 \%$ sont moyennement performantes et $35 \%$ ne le sont pas. Pour déterminer la proportion des propriétaires-dirigeants africains qui recourent à des pratiques de gestion, nous avons considéré les quatre activités suivantes : la planification, l'organisation, le contrôle et la direction. Au total, dix-huit pratiques de gestion ont été retenues, dont onze relatives à la planification, deux à l'organisation, quatre au contrôle et une à la direction. Lors du calcul, un point a été attribué à chaque pratique. Chaque pourcentage traduisant la proportion dans laquelle une entreprise recourt aux techniques de gestion correspond donc à la somme des points recueillis sur l'ensemble des pratiques considérées. Nous avons ainsi pu constater que $32 \%$ des répondants recourent à la planification, que $48 \%$ ont recours à l'organisation, que $79 \%$ ont un système de contrôle et que $21 \%$ des dirigeants prennent seuls les décisions. Bien que $79 \%$ des répondants affirment consulter une tierce personne (employée ou non), la décision finale est prise par le propriétairedirigeant lui-même. Des quatre activités de gestion considérées, on remarque que le contrôle constitue la préoccupation majeure des propriétaires-dirigeants congolais, suivi de la planification. L'organisation est également prise en compte, mais n'est en général pas très respectée. Les activités de direction entrent dans le domaine réservé du propriétaire-dirigeant. Les résultats obtenus pour les quatre activités de gestion correspondent à $35 \%$ d'utilisation.

À l'échelle nationale, la mauvaise gestion a des conséquences catastrophiques sur le plan économique. Étant donné que l'entreprise est la principale source de création d'emplois ainsi que des biens et services nécessaires à la population et que l'on attribue les principales causes de faillite à une gestion inappropriée, une hausse du taux de disparition des entreprises entraînerait une baisse de la production, une diminu- tion considérable du nombre d'emplois et par conséquent, une baisse de la demande, ce qui plongerait le pays dans une situation de pauvreté extrême. Les données tirées de la revue État du monde sur le taux de croissance annuelle du PIB sont très révélatrices de l'état actuel de l'économie congolaise.

La mauvaise gestion a des conséquences catastrophiques sur le plan économique. Étant donné que l'entreprise est la principale source de création d'emplois ainsi que des biens et services nécessaires à la population et que l'on attribue les principales causes de faillite à une gestion inappropriée, une hausse du taux de disparition des entreprises entraînerait une baisse de la production, une diminution considérable du nombre d'emplois et par conséquent, une baisse de la demande, ce qui plongerait le Congo dans une situation de pauvreté extrême.
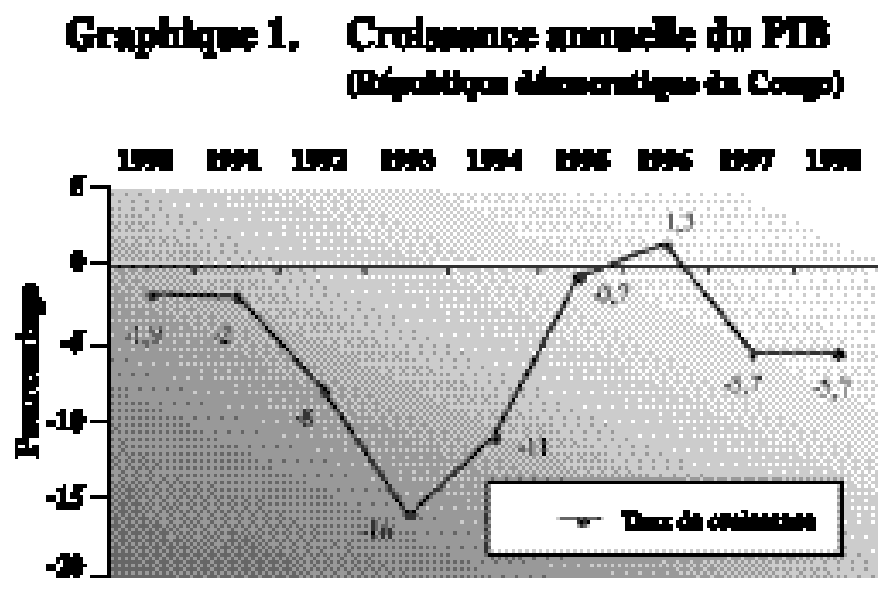

Arim

À la lueur de cette enquête, il apparaît sans aucun doute que les entreprises congolaises et africaines en général ont un besoin d'aide en matière de gestion et que le recours aux pratiques de gestion dans ces entreprises n'est pas chose courante. La recherche de solutions aux problèmes de gestion en Afrique ne date 
pas d'hier. Du point de vue environnemental, il faudrait créer un milieu propice à l'émergence, à la croissance et au développement des entreprises et qui contribuerait à rendre les pratiques de gestion des entreprises plus efficaces. Pour cela, il est capital d'amener les différents acteurs économiques à préciser entre eux, à travers un processus de négociation, les mesures et programmes économiques les plus susceptibles de favoriser l'accroissement de la richesse de tous les groupes. Il faudrait pour cela un engagement clair et ferme du gouvernement, en collaboration avec les autres partenaires, à appliquer les solutions envisagées. Il faut préciser qu'un environnement hostile a uniquement pour effet d'amoindrir l'efficacité des pratiques de gestion, contrairement aux valeurs et us, qui peuvent aussi agir sur leur utilisation. Si l'efficacité et l'utilisation des pratiques manageriales sont remises en question en Afrique en grande partie à cause des us et coutumes, la solution au problème ne peut être trouvée que dans les valeurs africaines. Il est donc impératif de procéder à la définition de la culture africaine, de tracer un profil général des entreprises de ce territoire pour identifier les éléments qui sont peu ouverts aux techniques manageriales, de détecter les différentes pratiques de gestion qui sont en contradiction avec les valeurs africaines en vue de les modifier si possible et de les rendre plus compatibles avec ces valeurs et les particularités des entreprises africaines. Un travail pourrait également être fait en ce qui a trait aux us et coutumes par la sensibilisation aux bienfaits d'un recours aux pratiques de gestion au sein des organisations en faisant l'éducation des masses. La réaction de certaines personnes pourrait être reten-
La recherche de solutions aux problèmes de gestion en Afrique ne date pas d'hier. Du point de vue environnemental, il faudrait créer un milieu propice à l'émergence, à la croissance et au développement des entreprises et qui contribuerait à rendre les pratiques de gestion des entreprises plus efficaces. Pour cela, il est capital d'amener les différents acteurs économiques à préciser entre eux, à travers un processus de négociation, les mesures et programmes économiques les plus susceptibles de favoriser l'accroissement de la richesse de tous les groupes.

tissante si des actions visant des changements culturels étaient posées. Il est vrai que la culture constitue l'identité d'un peuple et une richesse inestimable. Mais il faut reconnaître cependant que certaines de ces valeurs freinent le développement et que, si l'Afrique veut se développer, il faudrait peut-être envisager de changer certains de nos comportements.

\section{Note}

1 Chirushage Chirishungu est diplômée de $2^{\mathrm{e}}$ cycle en gestion des organisations de l'Université du Québec à Chicoutimi. 